\title{
Efecto del pastoreo sobre la productividad primaria neta subterránea de un pastizal templado
}

\author{
Luis López-Mársico" ${ }^{1,}$; Mariano Oyarzabal $^{2}$; Alice Altesor ${ }^{1} \&$ José M. Paruelo ${ }^{1 / 2}$ \\ ${ }^{1}$ Instituto de Ecología y Ciencias Ambientales, Facultad de Ciencias, Universidad de la República. Montevideo, Uruguay. \\ ${ }^{2}$ Laboratorio de Análisis Regional y Teledetección y Departamento de Métodos Cuantitativos y Sistemas de Información, \\ IFEVA/Facultad de Agronomía, Universidad de Buenos Aires/CONICET. Buenos Aires, Argentina.
}

\begin{abstract}
RESUMEN. El pastoreo afecta directamente a la parte aérea de la vegetación, e indirectamente a la subterránea. En los pastizales existe un conocimiento escaso del efecto del pastoreo sobre la productividad primaria neta subterránea (PPNS). Nuestro objetivo fue cuantificar el efecto del pastoreo sobre la PPNS hasta $25 \mathrm{~cm}$ de profundidad en un pastizal de la región centro-sur de Uruguay, en cuatro períodos de muestreo distribuidos uniformemente a lo largo de un año. Al inicio de cada período se realizaron cinco pozos cilíndricos de $5 \mathrm{~cm}$ de diámetro x $25 \mathrm{~cm}$ de profundidad en tres áreas clausuradas de entre 4 y 17 años de antigüedad, y en sus áreas pastoreadas adyacentes. Se colocaron mallas plásticas cilíndricas, se rellenaron con suelo libre de raíces, se extrajeron al cabo de tres meses y se fraccionaron en tres profundidades $(0-5,5-10$ y 10-25 cm). La PPNS anual para el perfil de $25 \mathrm{~cm}$ fue $41 \%$ mayor en las áreas pastoreadas $\left(505 \mathrm{~g} . \mathrm{m}^{-2}\right.$.año ${ }^{-1}$ ) que en las clausuradas (357 g. $\mathrm{m}^{-2}$.año ${ }^{-1}$ ). La PPNS anual disminuyó con la profundidad del suelo de manera similar en las áreas pastoreadas y clausuradas. La mitad de la PPNS anual de todo el perfil estuvo concentrada en las primeras dos fracciones (0-5 y 5-10 cm). Para el perfil completo de $25 \mathrm{~cm}$, la PPNS presentó una interacción pastoreo $\mathrm{x}$ período de muestreo marginalmente significativa, lo cual indica trayectorias diferentes de la PPNS. Nuestro estudio es uno de los pocos que evalúa el efecto del pastoreo sobre la PPNS en pastizales del Río de la Plata y es el primero que lo hace por medio de la técnica de mallas cilíndricas. Cuantificar el efecto del pastoreo sobre la PPNS permite entender la dinámica de secuestro de C en el suelo, un servicio ecosistémico de gran valor que aportan los pastizales.
\end{abstract}

[Palabras clave: pastoreo-clausura, secuestro de C, distribución de raíces, mallas cilíndricas, pastizales del Río de la Plata, Uruguay]

\begin{abstract}
AвSTRACT. Effect of grazing on below-ground net primary productivity of a temperate grassland. Grazing directly affects above-ground fraction of vegetation and indirectly the below-ground part. There is a poor knowledge of the effect of grazing on below-ground net primary productivity (BNPP) in grasslands. Our aim was to quantify the effect of grazing on BNPP up to $25 \mathrm{~cm}$ depth in a south-central Uruguayan grassland, in four sampling periods, distributed uniformly throughout a year. At the beginning of each period, five cylindrical holes of $5 \mathrm{~cm}$ diameter $\times 25 \mathrm{~cm}$ depth, in three exclosures sites aged between 4 and 17 years, and at their adjacent grazed areas, were performed. Ingrowth cores filled with free roots soil were placed in the hole, extracted after three months and divided in 3 depths $(0-5,5-10$ and 10-25 cm). For $25 \mathrm{~cm}$ profile, the annual BNPP was $41 \%$ higher in grazed areas $\left(505 \mathrm{~g} \cdot \mathrm{m}^{-2}\right.$.year $\left.{ }^{-1}\right)$ than in exclosures areas $\left(357 \mathrm{~g} \cdot \mathrm{m}^{-2}\right.$.year $\left.{ }^{-1}\right)$. The annual BNPP decreased with soil depth similarly at grazed and exclosures areas. Half of the annual BNPP of whole profile was concentrated in the two shallowest layers $(0-5$ and 5-10 cm). BNPP for the profile of $25 \mathrm{~cm}$ presented interaction grazing $x$ sampling periods marginally significant, indicating different trajectories of BNPP. This study is one of the few in the Río de la Plata grasslands that assesses the effect of grazing on BNPP and the first using ingrowth cores. Quantifying the effect of grazing on BNPP allows understanding the dynamics of $\mathrm{C}$ sequestration in the soil, an ecosystem service particularly valued in grasslands.
\end{abstract}

[Keywords: grazed-ungrazed, C sequestration, root distribution, ingrowth cores, Río de la Plata Grasslands, Uruguay]

Editor asociado: Pedro Laterra

luislopez@fcien.edu.uy
Recibido: 27 de julio de 2015

Aceptado: 4 de julio de 2016 


\section{INTRODUCCIÓN}

El pastoreo afecta de manera directa a la parte aérea de la vegetación e indirecta a la subterránea (Ferraro and Oesterheld 2002) a través de un conjunto de componentes que ocurren simultáneamente. Si bien la defoliación es el componente más estudiado (y muchas veces considerado como el más relevante), el pisoteo y la deposición de heces y orina también provocan cambios en la estructura y en el funcionamiento, tanto a nivel aéreo como subterráneo (Mikola et al. 2009; Lezama and Paruelo 2016). El efecto del pastoreo sobre la productividad primaria neta aérea (PPNA) ha sido muy estudiado en pastizales (Milchunas and Lauenroth 1993; McNaughton et al. 1996). En cambio, el efecto del pastoreo sobre la productividad primaria neta subterránea (PPNS) es relativamente menos conocido (Sims and Singh 1978; López-Mársico et al. 2015) y, por otro lado, su estimación depende de la técnica de muestreo utilizada (Milchunas 2009). A nivel global en pastizales, la respuesta de la PPNS al pastoreo no presenta una tendencia clara (Sims and Singh 1978; Gao et al. 2008; García-Pausas et al. 2011). Sin embargo, en pastizales que reciben una precipitación acumulada anual superior a $800 \mathrm{~mm}$ se observaron respuestas en su mayoría positivas del pastoreo sobre la PPNS (Sims and Singh 1978; Pucheta et al. 2004; Xu et al. 2014). Además, el pastoreo puede modificar la estacionalidad de la biomasa subterránea (Piñeiro et al. 2009; LópezMársico et al. 2015). La PPNS representa, en promedio, el 70\% de la PPN total (Hui and Jackson 2006), lo que constituye una de las determinantes principales del secuestro de C por parte de los pastizales (Piñeiro et al. 2010). Se ha observado también que la fracción más superficial explica una gran parte de la PPNS del perfil del suelo (López-Mársico et al. 2015). Por lo tanto, cuantificar el efecto del pastoreo sobre la PPNS en distintos períodos del año y profundidades permitirá entender mejor los controles de la dinámica de $\mathrm{C}$ en el suelo.

Por su extensión, los Pastizales del Río de la Plata ocupan una de las regiones más importantes de pastizales templados en el mundo (Dixon et al. 2014). La región abarca $\sim 700.000 \mathrm{~km}^{2}$ entre los $28^{\circ}$ y $38^{\circ}$ de latitud sur, y comprende el este de Argentina, el sur de Brasil y todo el territorio de Uruguay (Soriano et al. 1991). En la región centrosur, una de las regiones geomorfológicas de Uruguay (Panario 1988), el pastoreo por ganado doméstico promueve cambios en la estructura y el funcionamiento (Altesor et al. 2005, 2006; López-Mársico et al. 2015). A nivel aéreo, el pastoreo determina dos estratos de vegetación (Altesor et al. 2005): un estrato bajo ( $<10 \mathrm{~cm}$ de altura), denso, con una cobertura vegetal cercana a $100 \%$, dominado por gramíneas $\mathrm{C}_{4}$ con crecimiento postrado $\mathrm{y}$ hierbas arrosetadas, y un segundo estrato $(>20$ $\mathrm{cm}$ de altura), dominado por gramíneas poco palatables, arbustos y sub-arbustos (Soriano et al. 1991; Altesor et al. 2005). En cambio, en las áreas clausuradas al pastoreo ocurre una co-dominancia de gramíneas $\mathrm{C}_{3}$ y $\mathrm{C}_{4}$ con crecimiento erecto, las gramíneas postradas y las hierbas arrosetadas del estrato bajo disminuyen su cobertura en relación a las áreas pastoreadas, hay acumulación de broza y aumenta la riqueza y la cobertura de arbustos (Altesor et al. 2005, 2006). A nivel subterráneo, la biomasa en las áreas pastoreadas es mayor que en las áreas clausuradas debido principalmente a una concentración mayor en la fracción más superficial del suelo (López-Mársico et al. 2015). En cuanto al funcionamiento, la PPN, tanto aérea como subterránea, es mayor en las áreas pastoreadas que en las áreas clausuradas (Altesor et al. 2005; López-Mársico et al. 2015). En las áreas pastoreadas, la dominancia de gramíneas estivales explica el aumento observado en la PPNA (Altesor et al. 2005), mientras que a nivel subterráneo, la mayor tasa de recambio de la biomasa en la fracción más superficial del suelo explica la mayor PPNS observada, en comparación con las áreas clausuradas (López-Mársico et al. 2015).

En los pastizales de gran parte del mundo, incluyendo los de Uruguay, existe un conocimiento escaso del efecto del pastoreo sobre la estructura y el funcionamiento de la vegetación a nivel subterráneo. Recientemente se estudió, a lo largo de un período de crecimiento, el efecto del pastoreo sobre la dinámica de la biomasa subterránea y la PPNS anual mediante el método clásico de cosechas secuenciales de volúmenes de suelo (López-Mársico et al. 2015). Sin embargo, se desconoce la dinámica de la PPNS a lo largo del año y para distintas profundidades del suelo. El objetivo de este trabajo fue cuantificar el efecto del pastoreo sobre la PPNS hasta 25 cm de profundidad en un pastizal de la región centro-sur de Uruguay, en cuatro períodos de muestreo, a lo largo de un año. Se empleó una técnica de muestreo distinta a la utilizada en un trabajo reciente (López-Mársico et al. 2015). La hipótesis fue que los cambios, entre las áreas pastoreadas y clausuradas, en cuanto a 
cobertura del estrato herbáceo y a composición de especies y tipos funcionales de plantas, determinan diferencias en la PPNS en función de la profundidad del suelo y del período de muestreo. Las predicciones fueron: 1) la PPNS anual será mayor en las áreas pastoreadas que en las clausuradas, determinada en particular por incrementos en las estaciones más cálidas, y 2) la PPNS disminuirá con la profundidad del suelo, aunque de forma más acentuada en las áreas pastoreadas que en las clausuradas.

\section{MATERIALES y MÉTODOS}

\section{Sitio de estudio, diseño y procesamiento de las muestras}

El muestreo se llevó a cabo en un pastizal de la región geomorfológica centro-sur, cercano a la localidad de Ecilda Paullier, departamento de San José, Uruguay ( $\left.34^{\circ} 20^{\prime} S, 56^{\circ} 58^{\prime} \mathrm{O}\right)$. Los tipos de suelo predominantes en esta región son vertisoles y brunosoles desarrollados sobre sedimentos cuaternarios, superficiales o profundos. El relieve es ondulado (Panario 1988) y cubre 1.3 millones de ha, de las cuales $72 \%$ corresponde a pastizal natural (Baeza et al. 2011; Lezama et al. 2011). La precipitación total en el período en que se tomaron las muestras fue $1460 \mathrm{~mm}, 30 \%$ más alta que el promedio histórico de los últimos 40 años, mientras que la temperatura media mensual fue máxima en enero $\left(27^{\circ} \mathrm{C}\right)$ y mínima en julio $\left(4.7{ }^{\circ} \mathrm{C}\right.$ ) (INIA La Estanzuela 2010). Los muestreos fueron realizados en un sitio típico de la región centro-sur de Uruguay, que tuvo uso agrícola en algunos potreros hasta mediados de la década de los ochenta del siglo pasado, y luego el uso fue exclusivamente ganadería extensiva, en particular vacuna. Dentro de la matriz de pastizal con pastoreo moderado (0.7 UG) existen tres clausuras al ganado mediante alambrado. Las clausuras presentan una superficie de entre 500 y 1000 $\mathrm{m}^{2}$, están separadas entre 700 y 1200 m y su antigüedad al comienzo del muestreo oscilaba entre 4 y 17 años. Todas las áreas están ubicadas en una posición topográfica alta y la profundidad de suelo es mayor a $1 \mathrm{~m}$. Las tres áreas clausuradas y sus correspondientes pares adyacentes de pastizal pastoreado constituyeron las repeticiones verdaderas del tratamiento pastoreo debido a que para un rango amplio de edades de clausuras (3-11 años), que incluyen a las áreas de muestreo de este trabajo, la composición florística es bastante homogénea (Altesor et al. 2006; Lezama et al. 2014).

La aproximación para estimar la PPNS fue la de medir la biomasa de raíces que crecieron dentrodemallas cilíndricas cribadas enterradas en el suelo en un período aproximado de tres meses. El muestreo fue selectivo y realizado en cuatro períodos de muestreo durante un período total que abarcó 385 días entre abril de 2009 y mayo de 2010 (Tabla 1). Al inicio de cada período de muestreo (los períodos no se corresponden exactamente con las estaciones del año), en cada par clausura-pastoreo se seleccionaron áreas representativas de cada niveldel tratamiento pastoreo. Seseleccionaron parches homogéneos de vegetación herbácea, alejados al menos $1 \mathrm{~m}$ de los arbustos y $2 \mathrm{~m}$ del alambrado para reducir el efecto borde. En cada una de las áreas se realizaron cinco pozos de $25 \mathrm{~cm}$ de profundidad y $5 \mathrm{~cm}$ de diámetro con un taladro tipo sacabocado, que representaron las subrepeticiones. El número total de pozos fue de 120 (2 niveles de pastoreo $\times 4$ períodos de muestreo $\times 3$ repeticiones x 5 sub-repeticiones). En cada pozo se colocó una malla plástica cilíndrica de $5 \mathrm{~cm}$ de diámetro, $25 \mathrm{~cm}$ de profundidad y $1 \mathrm{~cm}^{2}$ de criba, que luego se rellenó con suelo tamizado de textura franco-arcillosa y libre de raíces. Se apisonó levemente y se regó por única vez con 100 mL de agua. Las mallas se retiraron al final de cada período de muestreo, luego de aproximadamente tres meses de haber sido colocadas (Tabla 1). En esa misma fecha, se hicieron 30 nuevos pozos, alejados al menos $1 \mathrm{~m}$ de los anteriores, donde se colocaron nuevas mallas y se repitió todo el proceso. Todas las mallas extraídas con suelo y raíces se conservaron en freezer a $-12{ }^{\circ} \mathrm{C}$ hasta su procesamiento.

Tabla 1. Períodos de muestreo abarcados entre la fecha de colocación y retiro de las mallas cilíndricas, la duración en días y la temperatura media de cada período.

Table 1. Sampling periods covered between the date of placement and removal of ingrowth cores, the duration in days and the mean temperature of each period.

\begin{tabular}{lccc}
\hline \multicolumn{1}{c}{ Período de muestreo } & Fecha de muestreos & Duración (días) & Temperatura $\left({ }^{\circ} \mathrm{C}\right)$ \\
\hline Otoño-invierno & $22 / 04 / 09-30 / 07 / 09$ & 99 & 16.7 \\
Invierno-primavera & $30 / 07 / 09-27 / 10 / 09$ & 89 & 13.0 \\
Primavera-verano & $27 / 10 / 09-30 / 01 / 10$ & 95 & 21.3 \\
Verano-otoño & $30 / 01 / 10-12 / 05 / 10$ & 102 & 19.3 \\
\hline
\end{tabular}


En el laboratorio, el contenido de cada malla se dividió en tres fracciones (0-5, 5-10 y 10-25 $\mathrm{cm}$ de profundidad), totalizando 360 unidades de muestreo (120 pozos x 3 fracciones). Para facilitar la separación de las raíces, cada unidad de muestreo se sumergió en agua durante $24 \mathrm{~h}$ y luego de sucesivas flotaciones se retuvieron las raíces a través de una columna descendente de tamices (2000, 1000 y $500 \mathrm{~nm}$ de criba) (Lauenroth and Whitman 1971). Para dispersar los restos remanentes de suelo adherido a las raíces se utilizó agua a presión en forma de lluvia. Se empleó una lupa binocular con el fin de identificar y separar las raíces de otros órganos vegetales. La biomasa de raíces fue secada en estufa durante 48 h a $70{ }^{\circ} \mathrm{C}$, y luego fue pesada.

\section{Análisis de datos}

La biomasa de las raíces que creció dentro de las mallas, dividida por la cantidad de días de cada período de muestreo, fue considerada una estimación de la PPNS para ese tiempo $\left(\mathrm{g} \cdot \mathrm{m}^{-2} \cdot \mathrm{dí}^{-1}\right)$. La suma de la PPNS de cada período de muestreo constituyó una estimación de la PPNS anual (g.m $\mathrm{m}^{-2}$ año $\left.{ }^{-1}\right)$. Se utilizaron modelos mixtos para analizar los datos considerando a los tratamientos: pastoreo (con dos niveles) y períodos de muestreo (con 4 niveles) como efectos fijos y a las repeticiones (3 sitios) y subrepeticiones (5 pozos por sitio) como efectos aleatorios. Se ajustaron modelos para la PPNS anual y de cada período de muestreo, para todo el perfil $(0-25 \mathrm{~cm})$ y para cada profundidad por separado $(0-5,5-10$ y $10-25 \mathrm{~cm})$.

\section{Resultados}

La PPNS anual del perfil completo (0-25 $\mathrm{cm})$ fue $41 \%$ mayor $(F=13.6 ; P=0.004)$ en las áreas pastoreadas $\left(505 \pm 70 \mathrm{~g} \cdot \mathrm{m}^{-2} \cdot \mathrm{anno}^{-1}\right)$ que en las clausuradas (357 \pm 71 g.m $\mathrm{m}^{-2}$.año $\left.{ }^{-1}\right)$. La PPNS anual disminuyó con la profundidad del suelo con un patrón similar en las áreas pastoreadas y clausuradas (Figura 1). La mitad de la PPNS anual de todo el perfil estuvo concentrada en las primeras dos fracciones $(0-5$ y 5-10 cm), tanto en las áreas pastoreadas como en las clausuradas. La PPNS anual en cada una de las tres fracciones fue superior en las áreas pastoreadas con respecto a las clausuradas. La PPNS fue $40 \%$ mayor en la fracción $0-5 \mathrm{~cm}$ ( $F=6.5 ; P=0.029), 52 \%$ mayor en la fracción $5-10 \mathrm{~cm}(F=10.5 ; P=0.009)$ y $36 \%$ mayor en la fracción 10-25 cm ( $F=8.8 ; P=0.014)$ (Figura 1).
La PPNS del perfil completo $(0-25 \mathrm{~cm})$ presentó interacción pastoreo $\mathrm{x}$ período de muestreo marginalmente significativa $(F=2.96$, $P=0.088$ ), indicando que la PPNS fue afectada diferencialmente por el pastoreo según el período de muestreo (Tabla 2). La PPNS aumentó más de tres veces entre el primer (otoño-invierno 2009) y el tercer período de muestreo (primavera 2009-verano 2010) en las áreas pastoreadas, mientras que aumentó dos veces entre el primer (otoño-invierno 2009) y el último período de muestreo (verano-otoño 2010) en las áreas clausuradas (Tabla 2).

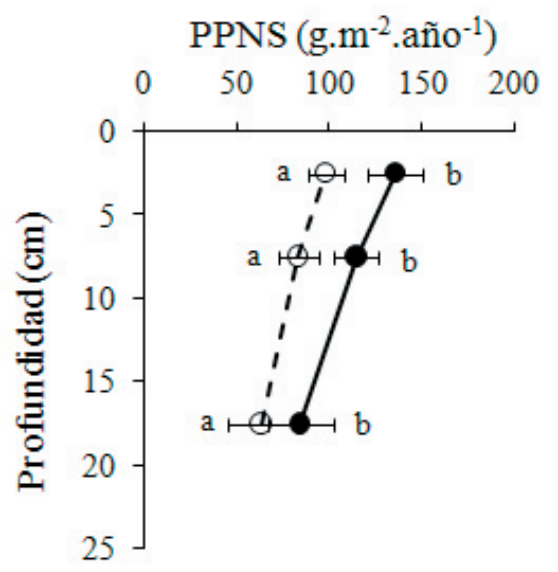

Figura 1. Productividad primaria neta subterránea (PPNS) anual para un perfil de $25 \mathrm{~cm}$ de profundidad de suelo en áreas pastoreadas (círculos negros) y clausuradas (círculos blancos) de un pastizal de la región centro-sur de Uruguay. Se presentan los valores promedio \pm DS $(\mathrm{n}=3)$. La PPNS de la fracción 10-25 cm corresponde a un tercio de la estimada. Los puntos se ubican en el medio de la fracción muestreada. Las letras distintas indican diferencias significativas con $P<0.05$ en cada fracción de suelo.

Figure 1. Annual below-ground net primary productivity (BNPP) of a $25 \mathrm{~cm}$ depth of profile soil in grazed and exclosures areas in a south-central Uruguay grassland. Mean values \pm SD $(n=3)$. BNPP of the layer $10-25$ corresponds to one third of the estimated. The points are located in the middle of the sampled layer. Different letters indicate significant differences with $P<0.05$ in each fraction of soil.

Tabla 2. Media \pm DS $(n=3)$ de la productividad primaria neta subterránea (PPNS en g.m. $\mathrm{m}^{-2} \cdot \mathrm{d}^{-1}$ ) para un perfil de 25 cm de profundidad, estimada a lo largo de cuatro períodos de muestreo en áreas pastoreadas y clausuradas de un pastizal de la región centro-sur de Uruguay.

Table 2.Mean \pm SD $(n=3)$ of below-ground net primary productivity (BNPP g. $\mathrm{m}^{-2} \cdot \mathrm{d}^{-1}$ ) for a profile of $25 \mathrm{~cm}$ depth estimated along four sampling periods in grazed and exclosures areas in a south-central Uruguayan grassland.

Áreas de muestreo

\begin{tabular}{lcc} 
Períodos de muestreo & Pastoreadas & Clausuradas \\
\hline Otoño-invierno & $0.55 \pm 0.17$ & $0.66 \pm 0.06$ \\
Invierno-primavera & $1.43 \pm 0.16$ & $0.93 \pm 0.20$ \\
Primavera-verano & $1.89 \pm 0.50$ & $1.11 \pm 0.46$ \\
Verano-otoño & $1.73 \pm 0.52$ & $1.39 \pm 0.63$ \\
\hline
\end{tabular}




\section{DISCUSIÓN}

El pastoreo por ganado doméstico promovió un aumento de la PPNS, tanto anual como en algunos períodos de muestreo en un pastizal de la región Centro-Sur de Uruguay, tal como se esperaba en la primera predicción. Esta diferencia se observó a lo largo de todo el perfil de suelo estudiado. A su vez, existió una disminución de la PPNS con la profundidad del suelo como esperábamos en nuestra segunda predicción, aunque de forma similar entre las áreas pastoreadas y clausuradas. Nuestras estimaciones de la PPNS anual están dentro del rango reportado para este tipo de ecosistemas (Hui and Jackson 2006; Paruelo et al. 2010). Tomando como referencia datos de PPNA de Altesor et al. (2005), para este mismo sitio de estudio, la relación PPNS/ PPN se encuentra cerca del límite inferior mencionado en la literatura (0.47; Hui and Jackson 2006) y no varió entre los tratamientos de pastoreo (0.46 y 0.48 en áreas pastoreadas y clausuradas, respectivamente). El efecto del pastoreo sobre la PPNS no presenta en pastizales de todo el mundo un patrón claro (Sims and Singh 1978; Gaoet al. 2008; GarcíaPausas et al. 2011) y los pastizales del Río de la Plata no son la excepción (Doll 1991; Soriano et al. 1991; López-Mársico et al. 2015). En la Pampa inundable existen, hasta donde sabemos, únicamente dos investigaciones que evaluaron el efecto del pastoreo sobre la PPNS y sus resultados fueron contradictorios, ya que en una la PPNS aumentó (Soriano et al. 1991) y en la otra disminuyó en respuesta al pastoreo (Doll 1991). Nuestros resultados presentan el mismo patrón observado en el mismo sitio de estudio pero en un año con precipitaciones inferiores al promedio histórico anual (López-Mársico et al. 2015). Independientemente de las precipitaciones, los resultados de un modelo aplicado para un promedio de 5 años muestran lo mismo, que la PPNS anual aumenta con el pastoreo (López-Mársico et al. 2015).

Las diferencias estructurales y funcionales observadas a nivel aéreo entre áreas pastoreadas y clausuradas (Altesor et al. 2005, 2006) reflejarían patrones similares a nivel subterráneo. Un análisis sucesional realizado en pastizales naturales de Uruguay reporta que el establecimiento de clausuras en áreas pastoreadas produce un recambio notable en la composición florística y en la distribución vertical de la biomasa vegetal al cabo de 2 ó 3 años (Rodríguez et al. 2003). En la región centro-sur no existen estudios que revelen el tiempo en que se generan estos cambios, aunque la composición florística para un amplio rango de edades de clausuras es bastante homogénea (Altesor et al. 2006; Lezama et al. 2014). Sin embargo, se ha notado en los pastizales pastoreados que la biomasa aérea se concentra en los primeros $10 \mathrm{~cm}$ del suelo debido a la presencia de especies con crecimiento postrado, mientras que en las áreas clausuradas se concentra en un estrato superior dominado por gramíneas erectas y arbustos (Altesor et al. 2005, 2006). Al igual que en la parte aérea, a nivel subterráneo el pastoreo favorece la concentración de la biomasa en los primeros centímetros del suelo, mientras que la exclusión del pastoreo promueve menor concentración relativa de biomasa en superficie y mayor en profundidad (LópezMársico et al. 2015). El efecto combinado de la defoliación y el pisoteo promueven un incremento en la riqueza, en la cobertura de especies y en la PPNA a nivel herbáceo en las áreas pastoreadas, en comparación con las clausuradas (Altesor et al. 2005; Lezama y Paruelo 2016). A nivel subterráneo, estos mismos componentes del pastoreo, sumados a la deposición de heces y orina, actuarían en el mismo sentido, promoviendo un incremento en la biomasa subterránea y en la PPNS en áreas pastoreadas con respecto a las clausuradas (López-Mársico et al. 2015). La proporción de la PPNS que es asignada a biomasa subterránea es uno de los principales factores que controlan la acumulación de C orgánico en el suelo (Piñeiro et al. 2010). En este sentido, los pastizales contribuyen a mantener la composición atmosférica ya que son capaces de secuestrar hasta el 98\% del C en la fracción subterránea (Hungate et al. 1997). Por lo tanto, es necesario fomentar un buen manejo del pastoreo para que los incrementos registrados en la biomasa subterránea y en su tasa de recambio, en comparación con las áreas clausuradas (López-Mársico et al. 2015), se traduzcan en un aumento en el secuestro de C atmosférico.

Las clausuras al pastoreo en pastizales de Uruguay también generan cambios microambientales que afectan la interacción planta-suelo (Fernández et al. 2014). Las clausuras promueven una disminución de la radiación que llega al estrato herbáceo, así como también de la velocidad del viento y de la temperatura del aire (Fernández et al. 2014). Esto podría ayudar a explicar los cambios asincrónicos entre la PPNA y la PPNS en áreas pastoreadas y clausuradas. En nuestro sitio de estudio, la PPNA estacional alcanza el pico en primavera y verano en áreas clausuradas 
y pastoreadas, respectivamente (Altesor et al. 2005), mientras que, de acuerdo a nuestros resultados, la PPNS estacional presenta un patrón inverso. Las clausuras presentan una mayor disponibilidad de agua en el suelo y una limitación por luz en los estratos inferiores (Fernández et al. 2014). La mayor cantidad relativa de especies con ciclo invernal en las áreas clausuradas (Altesor et al. 2006), con mayor PPNA relativa en primavera, permitiría asignar biomasa a la porción subterránea y, por lo tanto, lograr una mayor PPNS en verano-otoño. En las áreas pastoreadas, el recurso limitante sería el agua, por lo que la mayor presencia relativa de especies con ciclo estival (Altesor et al. 2005) explicaría un incremento de la PPNS en primavera-verano, de modo de obtener más recursos del suelo y generar mayor forraje en verano.

Existen pocos antecedentes del uso del método de las mallas cilíndricas en pastizales de Sudamérica (Soriano et al. 1987; de Wysiecki and Pérez 1994). Este es el primer estudio que se realiza con esta técnica para evaluar el efecto del pastoreo sobre la dinámica anual de la biomasa subterránea en pastizales del Río de la Plata. En el presente trabajo se consideraron raíces $2 \mathrm{~mm}$, sin diferenciar en tipos funcionales de plantas. Si bien podría esperarse que el mayor aporte esté dado por raíces de especies herbáceas, las raíces finas de los arbustos también podrían estar contribuyendo a la PPNS. Al igual que el resto de las técnicas de muestreo, el método de las mallas cilíndricas introduce distorsiones en los valores absolutos de la PPNS (Milchunas 2009) debido a que siempre se comienza con biomasa igual a cero. Las principales críticas a este método se deben a las diferencias entre el sustrato dentro de las mallas y el suelo que las rodea, lo cual determina cambios en la textura, en la resistencia a la penetración radical y en la competencia a nivel subterráneo (Neill 1992; Lauenroth 2000). Por el contrario, el método de las mallas cilíndricas presenta las ventajas de ser relativamente sencillo, se lo puede repetir en el espacio y en el tiempo con un grado bajo de destrucción, y permite evitar la diferenciación entre tejido vivo y muerto, pues el crecimiento corresponde al tiempo ocurrido entre muestreos (Neill 1992). Si bien las raíces finas pueden tener tasas de recambio elevadas, existen otros órganos subterráneos con mayor longevidad que aportan a la disponibilidad y al flujo de $\mathrm{C}$ del suelo y que no son considerados por esta metodología. Esto último podría explicar la baja proporción relativa de PPNS en los primeros $10 \mathrm{~cm}$ de suelo (50\%). En el mismo sitio de estudio, al contrastar los resultados obtenidos por el método de las mallas cilíndricas y el método clásico (López-Mársico et al. 2015) se notó que la fracción superficial $(0-10 \mathrm{~cm})$ dio cuenta de entre 60 y $70 \%$ de la PPNS de los primeros $30 \mathrm{~cm}$ de profundidad, en áreas clausuradas y pastoreadas, respectivamente. Las estimaciones de la PPNS obtenidas por el método de las mallas cilíndricas para un perfil de $0-25 \mathrm{~cm}$ reflejan un incremento de $22 \%$ en las áreas clausuradas y una disminución de $20 \%$ en las áreas pastoreadas respecto a los valores que obtuvieron López-Mársico et al. (2015) usando el método clásico para el perfil $0-30 \mathrm{~cm}$. Por lo tanto, hemos notado que existen otros factores propios de la metodología empleada que son necesarios tener en consideración, como es la ausencia de vegetación en la superficie de los cilindros, lo cual reduciría el aporte vertical de raíces hacia el interior de las mallas, particularmente en nuestro caso en las áreas pastoreadas.

En conclusión, este trabajo muestra que el pastoreo tiene un efecto positivo sobre la PPNS anual y que disminuye con la profundidad del suelo, independientemente del tratamiento de pastoreo. Para este año de muestreo existe una diferencia en la dinámica estacional de la PPNS entre las áreas pastoreadas y clausuradas. Los cambios observados en los distintos períodos de muestreo, revelan que el efecto promotor del pastoreo sobre la PPNS no es uniforme en el tiempo y tiende a aumentar en la estación más cálida. Finalmente consideramos que cuantificar el efecto del pastoreo sobre la PPNS ayuda a entender la dinámica de secuestro de $C$ en el suelo, un servicio ecosistémico particularmente valorado en los pastizales.

Agradecimientos. Queremos agradecer especialmente por su ayuda en el campo a: G. Fernández, F. Pezzani, A. Guido, A. Mello, S. Baeza, C. Bagnato, V. Cayssials, B. Costa, F. Gallego, E. Leoni y F. Lezama y por la valorable asistencia técnica en gabinete a M. Texeira y a A. Geymonat. Tres revisores anónimos y Pedro Laterra han contribuido significativamente para lograr una mejor versión de este trabajo. Agradecemos también a los dueños del Establecimiento El Relincho por las facilidades brindadas. Este trabajo fue parcialmente financiado por el Instituto Nacional de Investigación Agropecuaria (FPTA 175), por la Agencia Nacional de Investigación e Innovación (BE-POS-2009781), y por el Inter-American Institute for Global Change Research (IAI) CRN3095, este último apoyado por la US National Science Foundation (Subvención GEO-1128040). 


\section{REFERENCIAS}

Altesor, A., M. Oesterheld, E. Leoni, F. Lezama, and C. Rodríguez. 2005. Effect of grazing on community structure and productivity of a Uruguayan grassland. Plant Ecol 179(1):83-91.

Altesor, A., G. Piñeiro, F. Lezama, R. B. Jackson, M. Sarasola, and J. M. Paruelo. 2006. Ecosystem changes associated with grazing in subhumid South American grasslands. J Veg Sci 17(3):323-332.

Baeza, S., F. Gallego, F. Lezama, A. Altesor, and J. M. Paruelo. 2011. Cartografía de los pastizales naturales en las regiones geomorfológicas de Uruguay predominantemente ganaderas. Capítulo 2. Pp. 33-54 en: A. Altesor, W.Ayala and J. M.Paruelo (eds.).Bases ecológicas y tecnológicas para el manejo de pastizales. INIA. Montevideo, Uruguay.

Dixon, A. P., D. Faber-Langendoen, C. Josse, J. Morrison, and C.J. Loucks. 2014. Distribution mapping of world grassland types. J Biogeogr 41(11):2003-2019.

Doll, U. M. 1991. C-14 translocation to the below ground subsystem in a temperate humid grassland (Argentina). Pp. 350-358 en: B. L. McMichael and H.Persson (eds.). Plant roots and their environment. Elsevier Science Publishers.

Fernández, G., M. Texeira, and A. Altesor.2014. The small scale spatial pattern of $C_{3}$ and $C_{4}$ grasses depends on shrub distribution. Austral Ecol 39(5):532-539.

Ferraro, D. O., and M. Oesterheld. 2002. Effect of defoliation on grass growth. A quantitative review. Oikos 98(1): 125-133.

Gao, Y. Z., M. Giese, S. Lin, B. Sattelmacher, Y. Zhao, and H. Brueck. 2008. Belowground net primary productivity and biomass allocation of a grassland in Inner Mongolia is affected by grazing intensity. Plant Soil307(1):41-50.

García-Pausas, J., P. Casals, J. Romanyá, S. Vallecillo, and M. T. Sebastiá. 2011. Seasonal patterns of belowground biomass and productivity in mountain grasslands in the Pyrenees. Plant Soil 340(1):315-326.

Hui, D., and R. B. Jackson. 2006. Geographic and interannual variability in biomass partitioning in grassland ecosystems: a synthesis of field data. New Phytol 169(1):85-93.

Hungate, B. A., E. A. Holland, R. B. Jackson, F. S. Chapin, H. A. Mooney, and C. B. Field. 1997. The fate of carbon in grasslands under carbon dioxide enrichment. Nature 388(6642):576-579.

INIA La Estanzuela. 2010. Banco de datos agroclimáticos (1965-2010).

Lauenroth, W. K., and W. C.Whitman. 1971. A rapid method for washing roots. J Range Manage 24(4):308-309.

Lauenroth, W. K. 2000. Methods of estimating belowground net primary production. Pp. 58-71 en: O. E. Sala, R. B. Jackson, H. A. Mooney, and R. Howarth (eds.). Methods in ecosystem science. Springer, New York.

Lezama, F., A. Altesor, M. Pereira, and J. M. Paruelo. 2011. Descripción de la heterogeneidad florística de las principales regiones geomorfológicas de Uruguay. Pp. 15-32 en: A. Altesor, W. Ayala and J. M. Paruelo (eds.).Bases ecológicas y tecnológicas para el manejo de pastizales.INIA. Montevideo, Uruguay.

Lezama, F., S. Baeza, A. Altesor, A. Cesa, E. J. Chaneton, and J. M. Paruelo. 2014. Variation of grazing-induced vegetation changes across a large-scale productivity gradient. J Veg Sci 25(1), 8-21.

Lezama, F., and J. M. Paruelo. 2016. Disentangling grazing effects: trampling, defoliation and urine deposition. A Veg Sci 19(4), 557-566.

López-Mársico, L., A. Altesor, M. Oyarzabal, P. Baldassini, and J. M. Paruelo.2015. Grazing increases below-ground biomass and net primary production in a temperate grassland. Plant Soil 392(1):155-162

McNaughton, S. J., D. G.Milchunas, and D. A.Frank. 1996. How can net primary productivity be measured in grazing ecosystems? Ecology 77(3):974-977.

Mikola, J., H. Setälä, P. Virkajärvi, K. Saarijärvi, K. Ilmarinen, W. Voigt, and M. Vestberg. 2009. Defoliation and patchy nutrient return drive grazing effects on plant and soil properties in a dairy cow pasture. Ecol Monogr 79(2): 221-244.

Milchunas, D. 2009. Estimating root production: comparison of 11 methods in shortgrass steppe and review of biases. Ecosystems 12(8):1381-1402.

Milchunas, D. G., and W. K.Lauenroth. 1993. Quantitative effects of grazing on vegetation and soils over a global range of environments. Ecol Monogr 63(4):327-366.

Neill, C. 1992. Comparison of soil coring and ingrowth methods for measuring belowground production. Ecology 73(5):1918-1921.

Panario, D. 1988. Geomorfología del Uruguay. Facultad de Humanidades y Ciencias. Montevideo, Uruguay.

Paruelo, J. M., G. Piñeiro, G. Baldi, S. Baeza, F. Lezama, et al. 2010. Carbon stocks and fluxes in rangelands of the Rio de la Plata basin. Rangeland Ecol Manag 63(1):94-108.

Piñeiro, G., J. M. Paruelo, E. G. Jobbágy, R. B. Jackson, and M. Oesterheld. 2009. Grazing effects on belowground C and $\mathrm{N}$ stocks along a network of cattle exclosures in temperate and subtropical grasslands of South America. Global Biogeochem Cy 23(2):1-14.

Piñeiro, G., J. M. Paruelo, M. Oesterheld, and E. G. Jobbágy. 2010. Pathways of grazing effects on soil organic carbon and nitrogen. Rangeland Ecol Manag 63(1):109-119.

Pucheta, E., I. Bonamici, M. Cabido, and S. Díaz. 2004. Below-ground biomass and productivity of a grazed site and a neighboring ungrazed exclosure in a grassland in central Argentina. Austral Ecol 29(2):201-208.

Rodríguez, C., E. Leoni, F. Lezama, and A. Altesor. 2003. Temporal trends in species composition and plant traits in natural grasslands of Uruguay. J Veg Sci 14(3):433-440.

Sims, P. L., and J. S.Singh. 1978. The structural and function of ten western North American grasslands. III. Net primary 
productivity, turnover and efficiencies of energy capture and water use. J Ecol 66(2):573-597.

Soriano, A., R. J. C. León, O. E. Sala, R. S. Lavado, V. A. Deregibus, et al. 1991. Rio de la Plata grasslands. Pp. 367-407 en: COUPLAND, R. (ed.). Natural grasslands: introduction and western hemisphere. Elsevier, Amsterdam.

Soriano, A., R. A. Golluscio, and E. Satorre. 1987. Spatial heterogeneity of the root system of grasses in the Patagonian arid steppe. Bulletin of the Torrey Botanical Club 114(2):103-108.

De Wysiecky, M. L., And C. Pérez. 1994. Producción de raíces de dos pastizales pastoreados de la Sierras de la Ventana, Provincia de Buenos Aires, Argentina. Ecología Austral 4(2):95-99.

Xu, X., Y. Luo, Z. Shi, X.Zhou, and D. Li. 2014. Consistent proportional increments in responses of belowground net primary productivity to long-term warming and clipping at various soil depths in a tallgrass prairie. Oecologia 174(3):1045-1054. 